\title{
Botulinum toxin for cervical dystonia: addressing treatment failure and improving outcomes
}

\author{
Ashley B. Pena \\ Department of Neurology, Mayo Clinic, Jacksonville, Florida, USA
}

\begin{abstract}
Introduction. Cervical dystonia is a form of focal dystonia characterised by tilting and turning of the head and neck. This can cause significant disability in affected patients. Botulinum toxin injections are the mainstay of therapy. However, approximately $30 \%$ of patients discontinue treatment.
\end{abstract}

Clinical reflections. Tyślerowicz et al. have provided a comprehensive review of the factors contributing to treatment failure. Such factors include appropriate identification of dystonia patterns, accurate injection of muscles, and addressing non-motor features of cervical dystonia.

Clinical implications. A systematic approach is needed to identify and address the potentially modifiable factors that contribute to treatment failure.

(Neurol Neurochir Pol 2020; 54 (3): 218-219)

Cervical dystonia is a common form of focal dystonia. It can cause dystonic head tremor, impaired range of motion, and musculoskeletal pain, leading to significant functional disability for the patient [1].

Effective and well tolerated treatment options are therefore vital to preserve and restore functional status. Oral medications are notoriously ineffective in the treatment of cervical dystonia, and can present significant adverse effects. Botulinum toxin therapy has therefore become the mainstay of treatment due to its proven clinical efficacy and favourable side effect profile $[2,3]$. However, approximately $30 \%$ of patients discontinue treatment with botulinum toxin injections [4].

Tyślerowicz et al. have presented a review of potential pitfalls in the treatment of cervical dystonia with botulinum toxin. In addition to providing a detailed analysis of reasons for botulinum toxin treatment failure, they have discussed strategies for optimising treatment [5].

Assuring the correct diagnosis is the all-important first step in treating any neurological disorder. In the case of cervical dystonia, this includes assessing for mimics, identifying comorbid movement disorders, and fully addressing secondary causes of cervical dystonia. There are a number of movement disorders with similar phenomenology that can mimic cervical dystonia. These include tic disorders, particularly dystonic tics for which additional behavioural and pharmacological therapies may be beneficial $[6,7]$. Cervical dystonia can also be seen in the context of other movement disorders including Parkinson's Disease and atypical parkinsonian disorders, which present additional symptomatology and disease progression that may affect treatment response [8].

Successful treatment with botulinum toxin requires proper identification of the pattern of dystonia. The classification system used to identify dystonic movements impacts upon which muscles are then targeted with botulinum toxin [9]. Tyślerowicz et al. emphasise the utility of the Col-Cap scheme for identifying the components of dystonic posturing [5]. Dystonic movements can include turning and tilting of the head and neck in the lateral and anterior-posterior planes. A combination of these abnormal postures is often seen [10]. Utilising techniques such as EMG guidance to assure proper placement of botulinum toxin injections may assist in this approach. The therapeutic response may also be improved by appropriately escalating the dose and the distribution of botulinum toxin injections within the targeted muscles 
$[11,12]$. Proper counselling of patients is an essential complement to this optimisation process. Patients who are unaware of the potential for optimisation with repeated injections may wrongly perceive botulinum toxin treatment to be ineffective, and thus prematurely discontinue treatment $[4,13]$.

For patients who have had a suboptimal response to botulinum treatment over a long period, considerations may include immunity to botulinum toxin and complications of cervical dystonia such as muscle contracture and fibrosis [5].

A frequently overlooked component of treatment response is the contribution of non-motor features associated with cervical dystonia. These can include psychiatric disorders such as anxiety and depression, which can degrade quality of life and contribute to a subjective lack of improvement. Psychiatric symptoms appear to occur independently of the severity of motor symptoms, rather than as a consequence of motor symptoms $[14,15,16]$. As such, psychiatric symptoms do not demonstrate response to the treatment of motor symptoms [17, 18]. This suggests that the treatment of psychiatric comorbidities could play an important role in improving quality of life in patients with cervical dystonia.

Tyślerowicz et al. emphasise that a systematic approach is needed to examine the numerous potential contributors to botulinum toxin treatment failure [5]. Specific attention should be paid to addressing modifiable contributors.

It is important to optimise botulinum toxin treatment not only to prevent patient disability and improve quality of life, but also to maximise treatment efficacy prior to pursuing more invasive treatment options such as deep brain stimulation or selective denervation procedures.

In light of the wide array of contributors to botulinum toxin treatment failure outlined by this review, the development of a protocol-driven rehabilitative approach may be beneficial to augment the treatment of cervical dystonia.

\section{References}

1. Albanese A, Bhatia K, Bressman SB, et al. Phenomenology and classification of dystonia: a consensus update. Mov Disord. 2013; 28(7): 863-873, doi: 10.1002/mds.25475, indexed in Pubmed: 23649720.

2. Brans JW, Lindeboom R, Snoek JW, et al. Botulinum toxin versus trihexyphenidyl in cervical dystonia: a prospective, randomized, double-blind controlled trial. Neurology. 1996; 46(4): 1066-1072, doi: 10.1212/wnl.46.4.1066, indexed in Pubmed: 8780093.

3. Greene P, Kang U, Fahn S, et al. Double-blind, placebo-controlled trial of botulinum toxin injections for the treatment of spasmodic torticollis. Neurology. 1990; 40(8): 1213-1218, doi: 10.1212/wnl.40.8.1213, indexed in Pubmed: 2199847.

4. Jinnah HA, Comella CL, Perlmutter J, et al. Dystonia Coalition Investigators. Longitudinal studies of botulinum toxin in cervical dystonia:
Why do patients discontinue therapy? Toxicon. 2018; 147: 89-95, doi: 10.1016/j.toxicon.2017.09.004, indexed in Pubmed: 28888929.

5. Tyślerowicz M, Kiedrzyńska W, Adamkiewicz B, et al. Cervical dystonia - improving the effectiveness of botulinum toxin therapy. Neurol Neurochir Pol. 2020 [Epub ahead of print], doi: 10.5603/PJNNS. a2020.0021, indexed in Pubmed: 32285434.

6. Szejko N, Jakubczyk A, Dunalska A, et al. Dystonic tics in patients with Gilles de la Tourette syndrome. Neurol Neurochir Pol. 2019; 53(5): $335-$ 340, doi: 10.5603/PJNNS.a2019.0046, indexed in Pubmed: 31621889.

7. Tipton PW. Searching for tics. Neurol Neurochir Pol. 2019; 53(5): 315316, doi: 10.5603/PJNNS.a2019.0050, indexed in Pubmed: 31664709.

8. Yoon WT. Comparison of dystonia between Parkinson's disease and atypical parkinsonism: The clinical usefulness of dystonia distribution and characteristics in the differential diagnosis of parkinsonism. Neurol Neurochir Pol. 2018; 52(1): 48-53, doi: 10.1016/j. pjnns.2017.11.004, indexed in Pubmed: 29196058.

9. Jost WH, Biering-Sørensen Bo, Drużdż A, et al. Preferred muscles in cervical dystonia. Neurol Neurochir Pol. 2020 [Epub ahead of print], doi: 10.5603/PJNNS.a2020.0022, indexed in Pubmed: 32227332.

10. Finsterer J, Maeztu C, Revuelta GJ, et al. Collum-caput (COL-CAP) concept for conceptual anterocollis, anterocaput, and forward sagittal shift. J Neurol Sci. 2015; 355(1-2): 37-43, doi: 10.1016/j. jns.2015.06.015, indexed in Pubmed: 26088286.

11. Speelman JD, Brans JW. Cervical dystonia and botulinum treatment: is electromyographic guidance necessary? Mov Disord. 1995; 10(6): 802, doi: 10.1002/mds.870100619, indexed in Pubmed: 8750005.

12. Van Gerpen JA, Matsumoto JY, AhIskog JE, et al. Utility of an EMG mapping study in treating cervical dystonia. Muscle Nerve. 2000; 23(11): 1752-1756, doi: 10.1002/1097-4598(200011)23:11<1752::aid-mus12>3.0.co;2-u, indexed in Pubmed: 11054755.

13. Brashear A, Bergan K, Wojcieszek J, et al. Patients' perception of stopping or continuing treatment of cervical dystonia with botulinum toxin type A. Mov Disord. 2000; 15(1): 150-153, doi: 10.1002/1531-8257(200001)15:1<150::aid-mds1024>3.0.c0;2-x, indexed in Pubmed: 10634256.

14. Stamelou M, Edwards MJ, Hallett M, et al. The non-motor syndrome of primary dystonia: clinical and pathophysiological implications. Brain. 2012; 135(Pt 6): 1668-1681, doi: 10.1093/brain/awr224, indexed in Pubmed: 21933808.

15. Fabbrini G, Berardelli I, Moretti G, et al. Psychiatric disorders in adult-onset focal dystonia: a case-control study. Mov Disord. 2010; 25(4): 459-465, doi: 10.1002/mds.22983, indexed in Pubmed: 2010837.

16. Ben-Shlomo Y, Camfield L, Warner T, et al. ESDE collaborative group. What are the determinants of quality of life in people with cervical dystonia? J Neurol Neurosurg Psychiatry. 2002; 72(5): 608-614, doi: 10.1136/jnnp.72.5.608, indexed in Pubmed: 11971047.

17. Slawek J, Friedman A, Potulska A, et al. Factors affecting the health-related quality of life of patients with cervical dystonia and the impact of botulinum toxin type A injections. Funct Neurol. 2007; 22(2): 95-100, indexed in Pubmed: 17637212.

18. Berardelli I, Ferrazzano G, Pasquini M, et al. Clinical course of psychiatric disorders in patients with cervical dystonia. Psychiatry Res. 2015; 229(1-2): 583-585, doi: 10.1016/j.psychres.2015.07.076, indexed in Pubmed: 26239770. 